\title{
Antropología
}

\section{Autopercepción del bienestar del cuidador familiar del adulto mayor y características sociodemográficas ${ }^{\star}$}

\section{Self-Perception of the well-being of the family caregiver of the elderly and sociodemographic characteristics}

\section{Autopercepção do bem-estar do cuidador familiar do idoso e carac- terísticas sociodemográficas}

\begin{abstract}
${ }^{*}$ Este trabajo se deriva del proyecto Bienestar del cuidador familiar del adulto mayor: una perspectiva de género, con financiamaiento del Programa Integral de Fortalecimiento Institucional/2008 otorgado al Cuerpo Académico Salud y Comportamiento Humano de la Unidad Académica Multidisciplinaria Matamoros-UAT.
\end{abstract}

Dra. Aurora Félix Alemán ${ }^{1}$, Dra. Rosa Ma. Aguilar Hernández², Dra. Ma. de la Luz Martínez Aguilar³, Dra. Hermelinda Avila Alpirez ${ }^{4}$, Dra. Laura Vázquez Galindo ${ }^{5}$, Dr. Gustavo Gutiérrez Sánchez ${ }^{5}$

${ }^{1-5}$ Unidad Académica Multidisciplinaria Matamoros, Universidad Autónoma de Tamaulipas, México. Ave del Maestro y Marte s/n Col. Alianza. C. P. 87410, Matamoros Tamaulipas México. Tel. Fax (868) 8122716

Cómo citar este artículo en edición digital: Félix Alemán, A; Aguilar Hernández, R.Ma; Martínez Aguilar, Ma de la L; Avila Alpirez, H; Vázquez Galindo, L; , Gutiérrez Sánchez, G. (2013) Autopercepción del bienestar del cuidador familiar del adulto mayor y características sociodemográficas.. Cultura de los Cuidados (Edición digital) 17, 36. Disponible en: http://dx.doi.org/10.7184/ cuid.2013.36.06

Correspondencia: Aurora Félix Alemán Playa Miramar No. 22 e/Playa Mocambo y Priv. Ciro R. de la Garza. Col. Playa Sol. C. P. 87470. Matamoros, Tamaulipas México.Tel.(868)812-27-16 afelix@uat.edu.mx.

Recibido: 12/01/2013. Aceptado: 03/05/2013

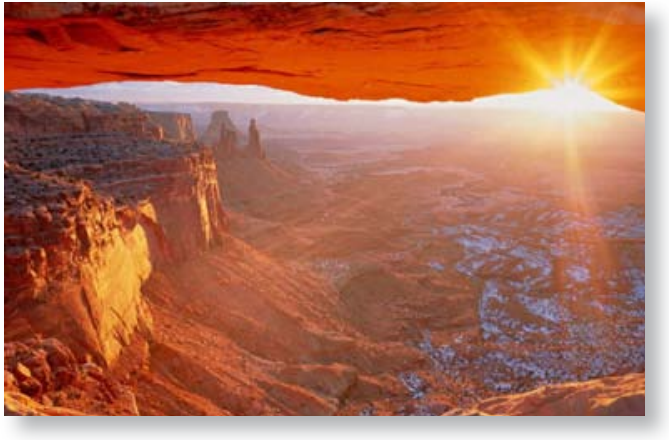

ABSTRACT

\section{Introduction}

Self-perception dimensional well-being is related to characteristics of caregivers.

\section{Objective}

To establish association of self-perception in dimensions of well-being and characteristics of caregivers.

\section{Method}

Descriptive, analytical and corelational study. Sample 300 caregivers. Matamoros Tamaulipas, México.

\section{Questionnaires}

Sociodemographic data, Barthel Index and Quality Life: dimensions physical, psychological, social; and spiritual, this, with alpha of Cronbach 0.69. Test Chi-square and Pearson's correlation coefficient.

\section{Results}

Features most them women, aged 42, married, daughter, literate, work home, low education levels, without work or income limited. Self-perception with significance general well-being, literacy and kinship; physical dimensions, age and gender; 
psychological, schooling, social, and spiritual kinship literacy, employment status, occupation and marital status.

\section{Discussion}

Characteristics harm to caregivers to adverse effects of care and determine greater perception of load that alters dimensional well-being.

Key Words: Self-perception, well-being, family caregiver, elderly

\section{RESUMO}

\section{Introdução}

A autopercepção do bem-estar dimensional relaciona-se com características de cuidadoras.

\section{Objetivo}

Estabelecer asociação de autopercepção em dimenções de bem-estar e características de cuidadores.

\section{Método}

Estudo descritivo, analítico e correlacional. Mostra 300 cuidadores. Matamoros Tamaulipas, México.

\section{Questionários}

Dados Sociodemográficos, Índice Barthel, Qualidade de vida: dimenções física, psicológica, social; e espiritual, este com alpha de Cronbach 0.69. Provas Qui-quadrado e coeficiente correlação de Pearson.

\section{Resultados}

Características maioria mulheres, 42 anos, casadas, filha, alfabetizadas, donas de casa, baixa escolaridade, sem emprego ou empregos limitados. Autopercepção com significância em bem-estar geral, alfabetização e parentesco; dimensões físicas, idade e gênero; psicológica, escolaridade; social, parentesco e espiritual alfabetização, situação de trabalho, ocupa e estado civil.

\section{Discusión}

Características vulneran a cuidadoras a efectos adversos del cuidado y determinan maior percepção de carga que altera seu bemestar dimensional.

Palavras chave: Autopercepção, bemestar, cuidador familiar, idoso.

\section{RESUMEN}

\section{Introducción}

La autopercepción del bienestar dimensional se relaciona con características de cuidadoras.

\section{Objetivo}

Establecer asociación de autopercepción en dimensiones de bienestar y características de cuidadores.

\section{Método}

Estudio descriptivo, analítico y correlacional. Muestra 300 cuidadores. Matamoros Tamaulipas, México.

\section{Cuestionarios}

Datos Sociodemográficos, Índice Barthel, Calidad Vida: dimensiones física, psicológica, social; y espiritual, éste con alpha de Cronbach 0.69. Pruebas chi-cuadrada y coeficiente correlación de Pearson.

\section{Resultados}

Características mayoría mujeres, 42 años, casadas, hija, alfabetizadas, labores hogar, baja escolaridad, sin trabajo o ingresos limitados. Autopercepción con significancia en bienestar general, alfabetización y parentesco; dimensiones física, edad y género; psicológica, escolaridad; social, parentesco y espiritual alfabetización, situación laboral, ocupación y estado civil.

\section{Discusión}

Características vulneran a cuidadoras a efectos adversos del cuidado y determinan 
mayor percepción de carga que altera su bienestar dimensional.

Palabras clave: Autopercepción, bienestar, cuidador familiar, adulto mayor.

\section{INTRODUCCIÓN}

Los adultos mayores son un difícil compromiso para el sistema de salud, debido a su declive biofisiológico y transformaciones de sus posiciones en las estructuras sociales, culturales y económicas

(Zabalegui, Rodríguez, Ramírez, Pulpón, López, Izquierdo, et al, 2007 \& Nigenda, LópezOrtega, Matarazzo, Juárez-Ramírez, 2007), ya que su condición de cronicidad y dependencia conlleva un cuidado sostenido que obliga a la familia a implicarse y le expone cambios que afectan su dinámica y relaciones interpersonales (Bover-Bover 2006). Entre las disciplinas de la salud, enfermería aborda el cuidado familiar desde la atención primaria con énfasis en grupos vulnerables como es el del adulto mayor cuya condición de dependencia amerita el cuidado de un familiar para atender sus necesidades. El cuidador familiar que cuida a un adulto mayor, está en condición de riesgo de perder su bienestar porque en la medida que cuida posterga sus necesidades (Velasco, 2010 \& Aldana-González, García-Gómez, 2011), con el agravante de falta de apoyo familiar ( $\mathrm{Za}$ balegui et al, 2007 \& Robles, Vázquez-Garnica, 2008) e inexistencia como sujeto del cuidado en instancias sociales (Administración Pública Federal. Consejo Nacional de Evaluación de la Política de Desarrollo Social, 2010) y de salud (Secretaría de Salud. Programa Nacional de Salud, 2007-2012).

La asunción del cuidado del adulto mayor sucede en un contexto de crisis familiar que finalmente recae en una persona con relación

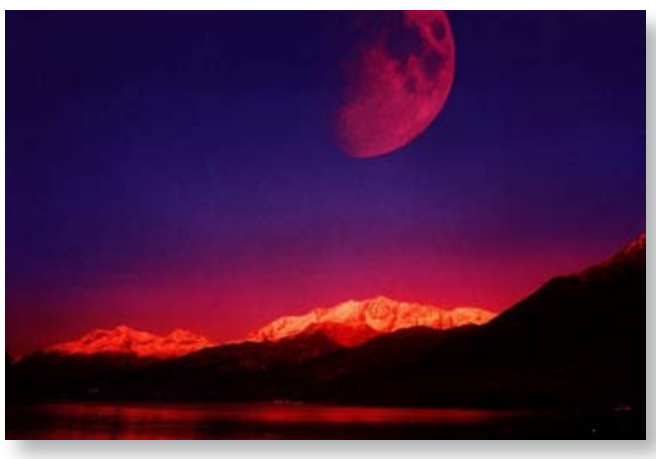

de parentesco, comúnmente esposa/o, hija/o (Bover-Bover, 2006; Robles, Vázquez-Garnica, 2008 \& Méndez-Luck, Kennedy, Wallace, 2008) denominado cuidador familiar que es definido como la persona,... atiende sus necesidades básicas de la vida diaria y lo apoya o supervisa en estas tareas; asume su cuidado de salud integral... sobre su cuidado" (Félix, Aguilar, Martínez, Avila, Vázquez, Gutiérrez, 2011) y que puede responder y adaptarse a las condiciones del cuidado de muy diversas formas (Zabalegui et al. 2007 \& Aldana-González, García-Gómez, 2011), razones y circunstancias, en cómo el cuidado se asume y se sostiene (Pessuto, Ferreira, 2008 \& Méndez-Luck et al., 2008). Si el cuidado se realiza con disposición, puede ser una experiencia gratificante y estimulante, ya que al cuidar se descubren cualidades, aptitudes y talentos mientras se convive con sentimientos como amor, cariño, agradecimiento y voluntad (Bover-Bover, 2006 \& Méndez- Luck et al., 2008).

Si el cuidado se realiza por imposición y con altas demandas de atención, limitaciones personales, económicas y de apoyo familiar, puede originar sentimientos ambivalentes como frustración, impotencia, desesperanza, angustia, tristeza, enojo y coraje que conduce a pérdida del bienestar (Robles-Silva, 2007 \& Méndez-Luck et al., 2008). La adaptación a la situación del cuidado determina la percepción que el cuidador familiar tenga de su bienestar. 
Lo que resulta del proceso de clasificar, interpretar, analizar e integrar las demandas del cuidado de manera que el cuidador familiar logre transformar y adaptar las circunstancias del mundo físico en la realidad psicológica (Feldman, 2006), que se traduce en la satisfacción de sus necesidades en forma holística: físicas, psicológicas, sociales y espirituales.

El Bienestar del Cuidador/a Familiar de Adultos Mayores con Dependencia Funcional se ha definido como la adaptación e integración del cuidador familiar, como ente holístico, en las dimensiones, física, psicológica, social y espiritual en el continuum salud-enfermedad" (Félix, Aguilar, Martínez, Avila, Vázquez Gutiérrez, 2012). El sentido de responsabilidad de los cuidadores familiares de adultos mayores los compromete a centrar en ellos la atención y los recursos (Dueñas, Martínez, Morales, Muñoz, Viáfara, Herrera, 2006) e incluso cuando los requerimientos del cuidado aumentan en exigencias y se prolonga en el tiempo, conlleva al autoabandono de proyectos, anhelos, motivaciones y en general del cuidado de su bienestar (Mier, Romeo, Canto, Mier R., 2007; DiGirolamo, Salgado, 2008; Roberto, Jarrott, 2008) así como la pérdida de sus redes sociales (Arroyo, Lancharro, Morillo, Romero, PérezGodoy, Moreno-Guerín, 2013), por lo que la autopercepción del bienestar puede ser en diferentes grados de intensidad.

Para lograr y mantener el bienestar en las cuatro dimensiones, los cuidadores de adultos mayores requieren tener habilidades para cumplir, independientemente de las circunstancias, con las demandas de su papel; en tanto que se compensen sus dificultades para satisfacer las necesidades físicas, psicológicas, sociales y espirituales. De ahí la importancia de considerar las definiciones que para este efecto se elaboraron.
Autopercepción del Bienestar es la satisfacción que manifiesta el cuidador/a familiar del adulto mayor en cuanto a la atención de sus necesidades como alimentación, eliminación, sueño, entre otras (Físico); amor, cariño, comprensión, apoyo, entre otras (Psicológico); relaciones personales, familiares, de pareja, laborales, financieros, actividades del hogar, entre otras (Social); valores, fe, esperanza, relación con un ser supremo, entre otras (Espiritual). Las necesidades que subyacen a la autopercepción pueden estar alteradas por el cumplimiento de su papel como cuidadoras. Quienes, sin embargo, pueden responder positivamente en la medida en que logren compensar los problemas derivados y afrontarlos (Pessuto, Ferreira, 2008).

El ser mujeres en su mayoría, como característica peculiar en el grupo de cuidadores familiares conlleva a la referencia en este estudio como "cuidadoras familiares". Su caracterización es que el cuidado les es socialmente asignado tanto en los países con alto nivel de desarrollo (Koerner, Kenyon, 2007; Vellone, Piras,Talucci, Cohen, 2008; Instituto de Mayores y Servicios Sociales. Cuidadores de Mayores, 2010 \& Yonte, Urién, Martín, Montero, 2010) como en países Latinoamericanos (Rojas, 2007; Espinosa, Mendez, Lara, Rivera, 2009) y en México (Zabalegui et al., 2007; Di Girolamo, Salgado, 2008 \& Lara, González, Blanco, 2008; Lagarde, 2011) con edad media de 45 años y mas (Nigenda et al., 2007; Lara et al.,2008; Rodríguez, Pascual, Abreu, 2008; Yonte et al., 2010 \& Velasco, 2010), hijas del adulto mayor en su mayoría casadas (Nigenda et al., 2007; Lara et al., 2008; Rodríguez et al. 2008; Espinosa et al. 2009 \& Yonte et al., 2010), dedicadas al hogar, de recursos limitados y de baja escolaridad (Nigenda et al., 2007 \& Yonte et al., 2010), la mayoría alfabe- 
tizadas (Espinosa et al., 2009; Velasco, 2010 \& Yonte et al., 2010) sin trabajo remunerado $\mathrm{y}$ con limitaciones para realizarlo (Nigenda et al., 2007; Rodríguez et al., 2008; Espinosa et al., 2009 \& Velasco, 2010). Se mantienen en el cuidado sin preparación, el que adquieren en condiciones de estrés (Nigenda et al., 2007; Robles, Vázquez-Garnica, 2008 \& Espinosa et al., 2009), como algo ineludible, como única opción y preferencia del adulto mayor de ser cuidado en el hogar (Nigenda et al., 2007) y en la disyuntiva de dar prioridad a la satisfacción de las necesidades del adulto en perjuicio de su salud (Velasco, 2010 \& Aldana-González, García-Gómez, 2011). La peculiaridad de las cuidadoras familiares de adultos mayores las convierte en sujetos en riesgo de perder el bienestar y pueden pasar de ser cuidadoras a ser pesonas con necesidades de recibir cuidado (Rojo-Pérez, Fernández-Mayoralas, Forjaz, Delgado-Sanz, Ahmed-Mohamed, MartínezMartín et al., 2008).

Los estudios que han abordado la situación de cuidadoras familiares en cuanto a los efectos adversos lo han hecho desde enfoques diversos: salud fisica y psicologica (Dueñas et al., 2006), sobrecarga (Velasco, 2010; Yonte et al., 2010; Espinosa et al., 2009) y morbilidad (Luengo, Araneda, López et al., 2010; Alpuche, Ramos, Rojas, Figueroa, 2008 \& Lara et al, 2008), cuyos resultados evidencia la afectación de la salud en las dimensiones fisica, psicológica y social sin considerar la espiritual como una dimensión importante, en algunos casos la consideran parte de lo psicológico o social.

Asimismo la autopercepción del bienestar en forma holística: física, psicológica, social y espiritual y su relación con sus características, no ha sido estudiada por profesionales de enfermería en México. En la práctica de enfermería el cuidado debe ser holístico, en éste; lo espiritual cobra importancia debido a la crisis existencial que por la enfermedad y el cuidado se vive (Beca, 2008; Sánchez-Herrera, 2009 \& Coward, 2010). Además “... las prácticas espirituales están relacionadas con una mejor salud mental, una mejor salud física y una mejor adaptación a la enfermedad y la discapacidad" (Pinedo, 2011), lo que anima a hacer frente a este desafío.

Se realizó el presente informe a partir de la pregunta de investigación ¿Cuál es la relación de la autopercepción del bienestar y las características sociodemográficas de la cuidadora familiar del adulto mayor de Matamoros Tamaulipas, México? El objetivo fue establecer la asociación de autopercepción en las dimensiones de bienestar y características de las cuidadoras familiares de adultos mayores; cuyos resultados servirán de base para planear el cuidado de enfermería en sus necesidades dimensionales que conlleve a mejorar su bienestar y la autopercepción positiva que le permita cuidar de su familiar adulto mayor.

\section{MATERIAL Y MÉTODO}

Estudio descriptivo, analítico y correlacional. Muestra por conveniencia de 300 cuidadores/as principales de un adulto mayor con dependencia funcional, residentes en diferentes sectores del área urbana de Matamoros Tamaulipas, México, mayores de 18 años, con más de seis meses de manera continua en el cuidado y sin pago.

Instrumentos: Cuestionario Datos Sociodemográficos y del Cuidado, elaborado ex profeso; Índice de Barthel, para medir la dependencia funcional del adulto mayor (CidRuzafa, Damián-Moreno, 1997), quienes encontraron alpha de Cronbach de 0.86 -0.92 y Calidad Vida QOL (por sus siglas en inglés), (Ferrell, Grant, 2010), con alpha de Cronbach 


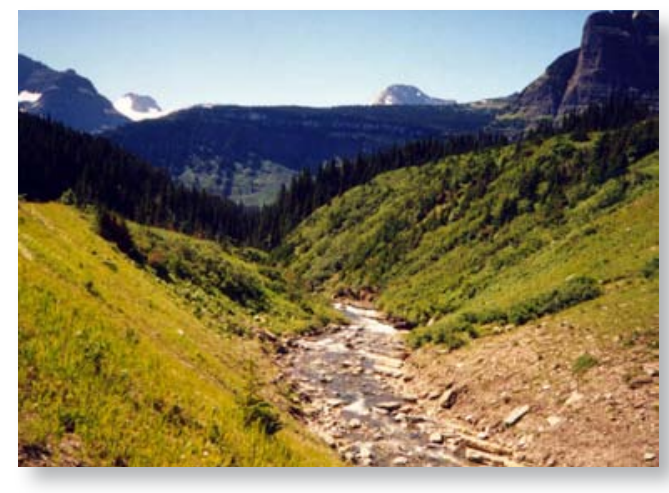

0.69. Es un instrumento ordinal, escala tipo Likert de 37 reactivos, organizados en cuatro secciones que corresponden a las dimensiones de la calidad de vida: física, psicológica, social y espiritual. Para este informe se consideró el último reactivo de cada sección que mediante su análisis permite identificar la autopercepción de la calidad de vida, el que se considera en este estudio como bienestar.

Procedimientos: se elaboró instructivo de cada instrumento para la capacitación y conducción de encuestadores: prestatario de servicio social y alumnos de la carrera de enfermería de la Unidad Académica Multidisciplinaria Matamoros, de la Universidad Autónoma de Tamaulipas (UAMM-UAT). La retroalimentación de la prueba piloto permitió uniformar la comprensión de las preguntas. Mediante visita domiciliaria se eligieron a cuidadores/ as con los criterios establecidos, previa firma del consentimiento informado para su participación. Para el procesamiento y análisis de los datos se utilizó el paquete estadístico SPSS, versión 15 , se aplicaron las pruebas estadísticas: chi-cuadrada y coeficiente de correlación de Pearson. El estudio se realizó apegado a lo dispuesto en el Reglamento de la Ley General de Salud en Materia de Investigación para la Salud (Secretaría de Salud, 1988) y con la aprobación del Comité de Investigación y Ética de la UAMM-UAT.

\section{RESULTADOS}

El grupo estudiado se caracterizó como: mujeres $(85.0 \%)$, en etapa media de la vida ( 42) o próximas a la etapa adulta mayor, casadas o en unión libre (65.0\%), hijo/a (61.4\%), alfabetizados (96.0\%), baja escolaridad ( 7.7), labores del hogar (66.3\%), sin actividad productiva $(49.3 \%)$ o con ingresos limitados (50.7\%). Se encontró significancia estadística en la autopercepción del bienestar en general de cuidadoras familiares de adultos mayores con la alfabetización y parentesco. La significancia de auto percepción en las dimensiones de bienestar del cuidador familiar del adulto mayor fue: en la física con edad y género, en la psicológica con escolaridad, en la social con parentesco y en la espiritual con alfabetización, situación laboral, ocupación y estado civil (Tabla 1).

\section{DISCUSIÓN}

El parentesco, principalmente las hija, son las que como asignación social asumen el cuidado y como lo refiere Lagarde (2011), es considerado una actividad propia de la mujer. Quienes son además, las preferidas por parte del adulto mayor para cuidarlos en el hogar, como encontraron Robles, Vázquez-Garnica (2008). Este componente relacional conlleva, como también encontraron, Aldana-González, García-Gómez (2011); Robles (2006 ), responsabilidad y fidelidad ineludible hasta el final del proceso de cuidado. La etapa media de la vida en la que se encuentran las cuidadoras conlleva cambios biológicos, psicológicos y sociales que ameritan autocuidado, lo cual postergan por el cuidado de su familiar, esto también fue encontrado por Velasco (2010); Aldana-González, García-Gómez (2011). Las cuidadoras del estudio, en su mayoria casadas, viven esta condicion con redes sociales: amis- 
tades, familiares o laborales, lo que alternan, por el cuidado, con un proceso de disolución de sí mismas, lo que tambien es referido por Aldana-González, García-Gómez (2011).

Las mujeres alternan su papel de cuidadoras con su ocupación en el hogar lo cual representa una doble jornada, hallazgo también referido por Méndez-Luck et al. (2008), lo que les limita para tener trabajo remunerado. El alto nivel de alfabetización de las cuidadoras del estudio les permite tener mayor conciencia en las circunstancias del cuidado, sin embargo el bajo nivel de escolaridad les impide o limita su percepción sobre las circunstancias del cuidado como las necesidades y gestión oportuna de apoyo que les facilite su papel como cuidadoras: comprensión de la enfermedad, aplicación de tratamientos, habilidades para el cuidado, técnicas de afrontamiento, entre otras; condición también encontrada por Del Prette, Del Prete (2002).

Estas características vulneran a las cuidadoras familiares de adultos mayores a los efectos adversos del cuidado y determina mayor percepción de la carga, así como es referido por BoverBover (2006); Méndez el al. (2008), dato similar al hallazgo de este estudio donde las cuidadoras familiares autoperciben alterado su bienestar físico, psicológico, social y espiritual. Circunstancia que en el contexto mexicano se acentúa debido al imperante sistema de organizacion familiar y del cuidado tipo patriarcal que establece para la mujer actividades del cuidado del hogar, la familia y de las personas dependietes; y a la falta de apoyo para el cudado: familiar, social y sanitario como lo refiere Nigenda et al. (2007). Lo anterior resta a las cuidadoras de adultos mayores oportunidades de desarrollo y crecimiento personal, social, laboral y profesional y lo más significativo, la autopercepción negativa de su bienestar.

\section{CONCLUSIÓN}

Las características de las cuidadoras familiares de adultos mayores resultaron asociadas en su mayoría con la autopercepción del bienestar espiritual y una o dos con las dimensione física, psicológica y social. Enfermería como profesión debe reconsiderar el cuidado holístico que involucra la dimensión espiritual y considerar a las cuidadoras familiares como un grupo vulnerable que requiere atención, de tal manera que les permita adaptarse a la dependencia de su familiar, afrontar la adversidad del cuidado y lograr la autopercepción física, psicológica, social y espiritual favorable y por efecto favorezcan y mantengan el bienestar del adulto mayor.

\begin{tabular}{|c|c|c|c|c|c|c|}
\hline \multirow{2}{*}{\multicolumn{2}{|c|}{ Autopercepción }} & \multirow{3}{*}{$\begin{array}{c}\begin{array}{c}\text { Salud } \\
\text { general }\end{array} \\
-\ldots--\end{array}$} & \multicolumn{4}{|c|}{ Dimensiones del bienestar } \\
\hline & & & Física & $\begin{array}{c}\text { Psicoló- } \\
\text { gica }\end{array}$ & Social & Espiritual \\
\hline Estado Civil & Casada & & -...- & -...- & -...- & 0.0353 \\
\hline Alfabetización & Alfabeta & 0.0344 & $\cdots$ & $\cdots$ & $\cdots$ & 0.0091 \\
\hline Ocupación & Hogar & -..-. & -..- & -.-- & $-\cdots$ & 0.0276 \\
\hline Situación Laboral & No trabajan & $\cdots$ & $\ldots$ & $\cdots$ & 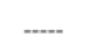 & 0.0200 \\
\hline Género & Femenino & 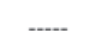 & 0.0209 & $\cdots$ & -..- & -..- \\
\hline Edad & $\bar{X} 42$ & $\cdots$ & 0.0003 & $\cdots$ & $\cdots$ & $\cdots$ \\
\hline Escolaridad & Baja & $\cdots$ & $\cdots$ & 0.0021 & $\cdots$ & $\ldots$ \\
\hline Parentesco & Hija & 0.0460 & $\cdots$ & -...- & 0.0820 & $\ldots$ \\
\hline
\end{tabular}

\section{BIBLIOGRAFÍA}

- Aldana-González, G. \& García-Gómez, L. (2011). La experiencia de ser cuidadora de un anciano con enfermedad crónica. Revista Aquichán, 11 (2), 158-72.

- Alpuche,VJ., Ramos, B., Rojas, ME. \& Figueroa, CG. ( 2008). Validez de la entrevista de carga de Zarit en una 
muestra de cuidadores primarios informales. Psicología y salud. 18 (2), 237-45.

- Administración Pública Federal. Consejo Nacional de Evaluación de la Política de Desarrollo Social. Programas por derechos sociales. 2010. Disponible en: <http://www.coneval.gob.mx/sipf//busquedas/busqueda.action?idDerBas=7> [Consultado el 8.05.2012].

- Arroyo, A., Lancharro, I., Morillo, Ma S., Romero, R., PérezGodoy. E. \& Moreno-Guerín, A. (2013). Etnografía del cuidador varón en una Unidad de lesionados medulares: análisis de los roles y redes sociales con una perspectiva de género. 17, 35. Disponible en: >http://dx.doi.org/10.7184/ cuid.2013.35.07> [Consultado el: 15.06. 2013].

- Beca, JP. (2008). El cuidado espiritual del enfermo como responsabilidad del profesional de la salud. Ética de los Cuidados. 1(1). Disponible en: <http://www. index-f.com/eticuidado/n1/et6734.php > [Consultado el 6.07.2012].

- Bover-Bover, A. (2006). El impacto de cuidar en el bienestar percibido por hombres y varones de mediana edad: una perspectiva de género. Enferm Clin, 16 (2), 69-76.

- Coward, D. (2010). Teoría de la Autotrascendencia En: Tomey, Ann Mariner; Raile Alligood Martha (6 $6^{\mathrm{a}}$ ed.). Modelos y Teorías de Enfermería (pp. 647-67). España. Elsevier Mosvy.

- Cid-Ruzafa, J. \& Damián-Moreno, J. (1997). Valoración de la discapacidad física: El índice de Barthel. Rev Esp Salud Pública, 71(2), 127-37.

- Dueñas, E., Martínez, MA., Morales, B., Muñoz, C., Viáfara, AS. \& Herrera, JA. (2006). Síndrome del cuidador de adultos mayores discapacitados y sus implicaciones psicosociales. Colomb Med. 37(2Suppl 1), 31-38.

- DiGirolamo, AM. \& Salgado, N. (2008). Women as primary caregivers in México: challenges to well-being. [Las mujeres como cuidadoras principales en México: retos para su bienestar]. Salud Pública Méx. 50 (6), 516-22.

- Del Prette, Z. \& Del Prete, A. (2002). Psicología de las habilidades sociales: terapia y educación. Manual Moderno, (2 $2^{\text {a }}$ ed.).

- Espinosa, E., Mendez,V., Lara, R. \& Rivera, P. (2009). Factores asociados al nivel de sobrecarga de los cuidadores informales de adultos mayores dependientes, en con- trol en el consultorio "Jose Durán Trujillo", San Carlos, Chile. Theoria. Revista Redaly 18(1):69-79.

- Félix, A., Aguilar, RM., Martínez, ML., Avila, H., Vázquez, L. \& Gutiérrez, G. (2011). Dependencia funcional del adulto mayor y caracterización del cuidador familiar. Paraninfo Digital. 2011. 5:14. Disponible en: <http://www.index-f. com/para/n14/013d.php > [Consultado el: 15.01. 2012].

- Félix, A., Aguilar, RM., Martínez, ML., Avila, H., Vázquez, L. \& Gutiérrez, G. (2011). Bienestar del cuidador/a familiar del adulto mayor con dependencia funcional: una perspectiva de género. Cul Cuid. XVI(33):81-88.

- Ferrell, B. \& Grant, M. (s.f). Quality of Life Family Version. National Medical Center and Beckman Research Institute. Disponible en : <http://prc.coh.org/pdf/ Quality of Life Family Version.pdf> [Consultado el 18.01.2010].

- Feldman, S. (2006). Psicología con aplicación en países de habla hispana. México. McGraw-Hill. (6 $6^{\mathrm{a}} \mathrm{ed}$.).

- Feldman, S. (2007). Desarrollo Psicológico a través de la vida. México. Pearson Educación. (4 $4^{\mathrm{a}}$ ed.).

- Instituto de Mayores y Servicios Sociales. Cuidadores de Mayores. La escasez de cuidadores informales, unido a la elevada demanda de asistencia, supone un amplio reto para los sistemas sociales y políticos (España). IMSERSO, 2010. Disponible en: <http://www.imsersomayores.csic.es/documentacion/especiales/cuidadores/ index.html> [Consultado el 22.02.2011].

- Koerner, S. \& Kenyon, B. (2007). Understanding “Good Days" and "Bad Days": Emotional and Physical Reactivity Among Caregivers for Elder Relatives. Family Relations. 56 (1):1-11.

- Lara, G., González, A. \& Blanco, LA. (2008). Perfil del cuidador: sobrecarga y apoyo familiar e institucional del cuidador primario en el primer nivel de atención. Rev Esp Med Quir. 13 (4):159-166.

- Luengo, CE., Araneda, G. \& López, MA. (2010). Factores del cuidador familiar que influyen en el cumplimiento de los cuidados básicos del usuario postrado. Index de Enfermería. 19(1):14-18.

- Lagarde, M. (2011). Los cautiverios de las mujeres: madresposas, monjas, putas, presas y locas. Madrid. horas y Horas. - Mendez-Luck, CA., Kennedy, DP. \& Wallace, SP. (2008). 
Concepts of Burden in Giving Care to Older relatives: A Study of Female Caregivers in a México City Neighborhood. J Cross. Cult Gerontol. 23:265-82.

- Mier, I., Romeo, Z., Canto, A. \& Mier, R. (2007). Interpretando el cuidado. Por qué cuidan sólo las mujeres y qué podemos hacer para evitarlo. ABENDUA, p. 29-38.

- Nigenda, G., López-Ortega, M., Matarazzo, C. \& JuárezRamírez, C. (2007). La atención de los enfermos y discapacitados en el hogar. Retos para el sistema de salud mexicano. Salud Publica Mex, 49(4): 286-94.

- Pinedo, MT. (2011). La humanización de los cuidados: reto de la enfermería delsiglo XXI. España. Consejo de Enfermería de la Comunidad Valenciana.

- Pessuto, J. \& Ferreira, C. (2008). Estratégias de coping desenvolvidas por cuidadores de idosos portadores de doença crónica. Rev Esc Enferm USP. 42(1):19-25. Disponible en: <www.ee.usp.br/reeusp/> [Consultado el: 13.12.2011].

- Robles-Silva, L. (2007). La designación de cuidadoras de padres enfermos: la última genitura femenina en un sector popular urbano de Guadalajara. En: Robichaux D., Editor. Familias mexicanas en transición: unas miradas antropológicas, p. 353-69. México Universidad Iberoamericana.

- Roberto, A. \& Jarrott, E. (2008). Family Caregivers of Older Adults: A LifeSpan Perspective. Family Relations.57(1): 100-111.

- Rojas, MV. (2007). Asociación entre la habilidad de cuidado del cuidador, el tiempo de cuidado y el grado de dependencia del adulto mayor que vive situación de enfermedad crónica, en la ciudad de Girardot. av. enferm. XXV (1):33-45.

- Rodríguez, M., Pascual, LM., \& Abreu, A. (2008). Relación de la alteración de las necesidades básicas de cuidadoras informales de ancianos dependientes y las dimensiones de calidad de vida (Huelva, España). Rev Paraninfo digital. 5 Disponible en: <http://www.indexf.com/para/n5/o053.php> [Consultado el 15.09.2011].

- Rojo-Pérez, F., Fernández-Mayoralas, G., Forjaz, Majo,, Delgado-Sanz, MaC., Ahmed-Mohamed, K. \& MartínezMartín, P. (2008). Población mayor, Calidad de Vida y redes de apoyo: demanda y prestación de cuidados en el seno familiar. Escuela Nacional de Sanidad, Instituto de Salud Carlos III-Ministerio de Ciencia e Innovación, 127p. Madrid. Disponible en: <http://www.isciii.es/ISCIII/es/ contenidos/fd-publicaciones-isciii/fd- documentos/Poblacion_mayor.pdf> [Consultado el 6.08.2011].

- Robles, L. \& Vázquez-Garnica, EK. (2008). El cuidado a los ancianos: las valoraciones en torno al cuidado no familiar. Texto contexto enferm. 17(2):225-31.

- Robles, L. (2006). El cuidado a los ancianos: la feminización de la obligación filial. En: Coordinadores. Robles, L., Vázquez, F., Reyes, L., Orosco, I. Miradas sobre la vejez. Un enfoque antropológico. México: Ed. Plaza y Valdez, 2006 p. 247-98.

- Secretaría de Salud. Programa Nacional de Salud (20072012). Por un México sano: Construyendo alianzas para una mejor salud. Disponible en: <http://www.conadic. salud.gob.mx/pdfs/programas/pns_version_completa. pdf> [Consultado el 8.07.2012].

- Sánchez, B. (2009). Bienestar espiritual en personas con y sin discapacidad. Revista Aquichán. 1):8-22

- Secretaría de Salud. Reglamento de la Ley General de Salud en materia de investigación para la salud México. Secretaría de Salud, 1988. Disponible en: <http://www. salud.gob.mx/unidades/cdi/nom/compi/rlgsmis.html> [Consultado el 18.01.2012].

- Velasco, A. (2010). Cuidador familiar del adulto mayor. Características y perfil de salud. de Portales Médicos [Revista Electrónica] 5:21. Disponible en: <http://www. portalesmedicos.com/publicaciones/articles/2598/1/ Cuidador-familiar-del-adulto-mayor-Caracteristicas-yperfil-de-salud.html> [Consultado el 11.02.2012].

- Vellone, E., Piras, G., Talucci, C. \& Cohen, MZ. (2008). Quantity of life for caregivers of people with Alzheimer's disease. J Adv Nurs.61(2): 222-31.

- Yonte, F., Urién, Z., Martín M. \& Montero, R. (2010). Sobrecarga del cuidador principal. Nure Investigación. 48:1-7.

- Zabalegui, A., Rodríguez, E., Ramírez, AM., Pulpón, A., López, L., Izquierdo, MD. et al. (2007). Revisión de evidencias: cuidadores informales de personas mayores de 65 años. Evidentia, 4(13). Disponible en <http://www.index-f.com/evidentia/n13/291articulo.php $>$ [Consultado el 15.05.2012]. 